\title{
Corela
}

Cognition, représentation, langage

HS-15 | 2014

Complémentarité des approches qualitatives et quantitatives dans l'analyse des discours

\section{Introduction : articuler les approches qualitatives et quantatives dans l'analyse de discours.}

\section{Thierry Guilbert}

\section{(2) OpenEdition}

Journals

Édition électronique

URL : http://journals.openedition.org/corela/3545

DOI : $10.4000 /$ corela. 3545

ISSN : 1638-573X

Éditeur

Cercle linguistique du Centre et de l'Ouest - CerLICO

Référence électronique

Thierry Guilbert, «Introduction : articuler les approches qualitatives et quantatives dans l'analyse de discours. », Corela [En ligne], HS-15 | 2014, mis en ligne le 15 octobre 2014, consulté le 01 mai 2019 URL : http://journals.openedition.org/corela/3545; DOI : 10.4000/corela.3545

Ce document a été généré automatiquement le 1 mai 2019.

\section{cc) (†) ()}

Corela - cognition, représentation, langage est mis à disposition selon les termes de la licence Creative Commons Attribution - Pas d'Utilisation Commerciale - Partage dans les Mêmes Conditions 4.0 International. 


\title{
Introduction : articuler les approches qualitatives et quantatives dans l'analyse de discours.
}

\author{
Thierry Guilbert
}

1 La thématique traitée dans ce numéro s'inscrit, d'un point de vue macroscopique, dans le cadre de la « révolution numérique » et de l'intégration de l'outil informatique dans notre vie quotidienne, l'ordinateur assistant de plus en plus nos tâches spécialisées - et pas uniquement dans le monde du travail ${ }^{1}$. On assiste ainsi dans le champ des sciences humaines et sociales et depuis une dizaine d'années, en France comme dans le monde francophone, à une véritable explosion de l'utilisation de logiciels ayant pour objet d'assister les chercheurs dans l'analyse des textes et des discours (ADT). La diffusion ou l'utilisation de ces logiciels n'est pas propre, comme on aurait pu s'y attendre au vu de son histoire, à l'analyse du discours (AD), ni même aux sciences du langage, elle traverse l'ensemble des sciences humaines qu'il s'agisse de l'histoire, de la psychologie sociale, de la sociologie, des sciences politiques, des sciences de l'information et de la communication ou encore des sciences juridiques.

2 Il est à noter que l'utilisation massive de ces logiciels s'accompagne d'un retour réflexif renouveléz à propos des méthodologies en sciences humaines et sociales. L'actualité de la recherche montre surtout un intérêt particulier pour les relations entre les approches qualitatives et quantitatives. Ainsi, par exemple, la revue Langage \& société consacre en 2011 un numéro aux méthodes quantitatives et qualitatives d'analyse des discours, Josiane Boutet et Didier Demazières saluent d'ailleurs dans leur propos introductif « cet intérêt croissant des chercheurs pour les discussions et réflexions méthodologiques " (2011 : 5). La même année, le numéro 55 de la revue Tranel, dirigé par Alain Kamber, est intitulé «La linguistique de corpus - de l'analyse quantitative à l'interprétation qualitative » et François Rastier publie La mesure et le grain, ouvrage qui traite justement des relations entre analyse quantitative et qualitative. On peut encore citer parmi les 
travaux récents, le numéro 187 de la revue Langages "L'analyse de corpus face à l'hétérogénéité des données" dirigé par Nathalie Garric et Julien Longhi paru en septembre 2012, la journée d'étude « Usages de la lexicométrie en sociologie » du 12 juin 2013, organisée à Versailles par le laboratoire Printemps (UMR 8085), et le colloque international «Complémentarité des approches qualitatives et quantitatives dans l'analyse des discours ? ", organisé à Amiens (UPJV) les 10 et 11 mai 2012 par le CURAPPESS (UMR 7319).

Ces journées amiénoises, qui ont permis de confronter les positionnements de chercheuses et chercheurs venant de disciplines et d'horizons différents, donnent lieu aux contributions qui composent ce volume. Celui-ci n'est cependant pas une simple compilation de communications rédigées mais un numéro de revue collectif et interdisciplinaire dont l'ambition est de présenter une cohérence scientifique et thématique : l'objectif commun est à la fois de penser le dépassement de l'opposition entre le qualitatif et le quantitatif et de souligner l'intérêt qu'il y a à penser leur articulation dans le contexte de la recherche actuelle et des outils mis à la disposition des chercheurs. Il ne s'agit pas, naturellement, de proposer une réponse unique. Les auteur-es ont accepté au contraire de discuter cette opposition/complémentarité, selon leur expérience, leur ancrage théorique dans leur pays d'origine (Brésil, Canada, France), leur formation et leur discipline (acquisition du langage, analyse du discours, histoire du discours politique), et de montrer comment, par un travail réflexif sur leur propre pratique scientifique - qu'ils utilisent ou non les méthodes assistées par ordinateur -, ils se situent vis-à-vis de ces questionnements. La cohérence de ce volume se manifeste donc dans la volonté partagée de dépasser l'opposition des deux approches, mais il revient au lecteur le soin de tirer ses propres conclusions.

4 Afin de problématiser quelque peu ces questions et de présenter les contributions, quatre remarques générales seront d'abord formulées quant à l'attrait pour ces logiciels et à la coexistence contemporaine du numérique, du numéral et des études sur le langage. Seront ensuite précisées un certain nombre de questions que pose actuellement à l'AD l'utilisation, hors des sciences du langage, voire parfois au sein des sciences du langage, des logiciels d'ATD. Les difficultés quant aux dénominations qualitatif/quantitatif ne seront ensuite qu'abordées - les contributions traitant amplement de ces questions. Enfin, cette introduction présentera brièvement les réflexions d'ordre méthodologique menées par les auteur-e-s dans ce volume.

\section{Le numérique, le numéral et le discours : de la logosphère à l'univers numéral ?}

5 Dès lors que l'on accepte le constat général de l'utilisation massive des logiciels d'analyse de textes, il semble qu'au moins quatre remarques s'imposent.

6 Premièrement, on sait depuis Marshall Mc Luhan que «les milieux ne sont pas des contenants passifs mais des processus actifs» (1976: 12), autrement dit que toute apparition et utilisation massive d'un nouveau médium modifie les relations entre les humains et leur rapport au monde, et ce jusque dans leur façon de s'exprimer (Gadet 2010 : 118-119). Pour ce qui nous concerne ici, la diffusion et l'utilisation des logiciels dans l'ATD modifient principalement le rapport qu'entretient le scientifique à la fois avec son objet et avec le langage. Ce questionnement est abordé dans la contribution présente de 
Marie-Anne Paveau de cette façon: «Comment se comporte l'alternative qualitatif/ quantitatif dans l'étude des discours natifs en ligne qui posent à la linguistique le problème de leurs caractéristiques technolangagières et technodiscursives?».

7 La deuxième remarque concerne les modifications de notre rapport à nos objets et au langage. Ces logiciels proposent ainsi un retour au langage comme moyen d'accéder au fonctionnement psychologique, sociologique, anthropologique, politique, etc., des acteurs, que ce soit aux plans cognitif, social ou communicationnel. Le désir manifeste d'analyser les discours, dans les travaux de chercheurs de disciplines et d'horizons différents, est l'indice d'une double volonté, à savoir analyser totalement les discours c'est la promesse de la plupart des logiciels (voir le logiciel Alceste in Dalud-Vincent 2011) - et faire du langage un élément essentiel de compréhension du fonctionnement des acteurs - c'est dans cette seconde perspective que s'inscrivent les sciences sociales. Si l'étude des discours n'a jamais été l'apanage de l'AD, voir se multiplier aujourd'hui des études sur le discours, hors de l'AD, notamment grâce à la diffusion et à la qualité des outils informatiques, ne peut que réjouir l'analyste du discours qui, il y a quelques années encore, bien après le reflux de la vague structuraliste, pouvait avoir l'impression, à tort ou à raison, qu'il était seul à en saisir l'importance. Ce phénomène devrait cependant lui poser question : que reste-t-il alors de la spécificité de l'AD aujourd'hui?

Troisièmement, force est aujourd'hui de constater une appétence généralisée pour le chiffre, le numéral. Signe de modernité et/ou de précision scientifique pour les uns ${ }^{3}$, signe de scientificité cosmétique ou de croyance dans les chiffres pour les autres, le numéral est maintenant présent dans des domaines où jamais auparavant on ne se serait attendu à le trouver ${ }^{4}$. Ce constat n'est pas sans poser un certain nombre des questionnements que je livre ici pêle-mêle. Même si cet attrait pour le numéral règne actuellement dans les sciences humaines et sociales, il est tout de même permis de se demander s'il s'agit toujours d'une recherche de la mesure, c'est-à-dire du quantifiable, plutôt que d'une recherche du chiffre, c'est-à-dire du quantitatif pour le quantitatif. On peut se demander également si tout phénomène social est quantifiable ou mesurable, autrement dit si la toise fonctionne vraiment dans et pour tous les domaines ou si le chercheur ne se fait pas Procuste quelquefois en redimensionnant les phénomènes à la mesure du lit de fer qu'est le cadre méthodologique numérique qu'il s'est fixé. On retrouvera ces questionnements dans les contributions de Jules Duchastel et Danielle Laberge, en analyse du discours, et d'Alessandra Del Ré et Rosângela Nogarini Hilário, en acquisition du langage. Enfin, et plus largement encore, l'imposition des mathématiques, donc du chiffre, dans l'économie à la fin des années 70 à Chicago (Dezalay \& Garth 1998), suivie de l'expansion des dogmes de l'économie à toutes les sphères de la vie sociale dans les années 80 et 90 (Vilette 2001), n'impose-t-elle pas la norme du chiffre - une «mathématisation du monde social» (Ogien 2013) -, jusque dans les laboratoires de sciences humaines et sociales?

9 La quatrième remarque interroge le sens et les conséquences du "réflexe quantitatif » que l'on rencontre quelquefois dans l'ATD : «ce qui importe c'est d'accéder au sens du message à travers les nombres ", rappellent ici J. Duchastel et D. Laberge à propos des méthodes quantitatives anglosaxonne et française. Ainsi recourir systématiquement aux quantités chiffrées laisse supposer que les approches qualitatives, quel que soit leur domaine, ne suffisent plus. Cette volonté - qui ressemble quelquefois à une injonction d'analyser totalement un corpus impose le recours aux traitements automatisés et semble discréditer, au moins en partie, les approches uniquement qualitatives. Il est vrai que, par 
définition, les secondes ne peuvent parvenir au degré de précision des premières pour ce qui concerne le quantifiable et que l'analyse de textes assistée par ordinateur présente l'intérêt d'être un outil heuristique en faisant découvrir au chercheur ce qu'il ne cherchait pas forcément (Mayaffre 2002). Dans sa contribution présente, Damon Mayaffre y revient en montrant précisément comment l'outil informatique propose au chercheur des pistes de réflexion. Si cette évolution est indéniablement un plus, c'est-à-dire un moyen supplémentaire d'accéder à la fois au sens et à la réalité des phénomènes observés, le «travail à la main » (Née \& Viénard 2012), «à l'humain » (M.-A. Paveau infra), doit-il aujourd'hui être forcément accompagné de ou assisté par l'outil informatique? L'« outillage quantitatif permet-il une plus grande vérité et validité ?», se demande ainsi M.-A. Paveau. Ces questions sont traitées à plusieurs reprises dans ce volume et les réponses apportées, à l'instar de Francine Mazière et Jacques Guilhaumou (2010), ne cherchent pas forcément à trancher pour l'une ou l'autre approche: "Les analyses les plus hardies semblent alors paradoxalement celles qui, faisant fi du quantitatif, osent le micro corpus qualitatif (Lucas Greco, Eni Orlandi) ou celles qui osent le parallèle dérangeant des résultats d'hier et d'aujourd'hui (Damon Mayaffre). Dans les deux cas, il faut des analystes aguerris, inventifs et/ou soutenus » $(2010: 80)$.

\section{Analyses quantitatives des discours et apports théoriques de l'AD}

10 La coexistence des approches qualitatives et quantitatives dans les sciences humaines et sociales et les questions relatives aux places respectives des deux approches ne sont pas nouvelles et ont donné lieu à des débats quelquefois houleux en histoire, comme le rappelle Serge De Sousa dans sa contribution située en histoire des discours politiques. Des discussions scientifiques fortes ont également marqué les sciences sociales, comme le montrent J. Duchastel et D. Laberge. L'analyse du discours n'a pas été épargnée par les heurts lors du développement conjoint, dès les années 60 , de ces deux approches.

11 Ainsi, au moment où se constituaient les « linguistiques de la parole » (Pêcheux 1975), c'est-à-dire les approches pragmatico-énonciatives sur le langage et son utilisation (à partir des travaux de Benveniste et de Culioli notamment), Michel Pêcheux concevait un dispositif critique d'analyse automatique du discours (AAD) à partir de la psychologie sociale (Léon 2010). A la même époque on assistait à l'introduction en France par l'université de Rouen de la sociolinguistique (qualitative) et à la naissance des premiers travaux de l'équipe de l'ENS de Saint-Cloud qui développait, autour de Maurice Tournier et Jean Dubois, une analyse quantitative du vocabulaire politique en créant ses propres outils lexicométriques. S. De Sousa y revient d'ailleurs dans sa contribution. Les outils se sont ensuite diversifiés en de nombreux travaux et logiciels quantitatifs (Muller, Tournier, Lafon, Lebart et Salem, Brunet) : lemmatiseurs, analyseurs syntaxiques, calculs statistiques, analyses des contextes et des spécificités, etc. (Cordial, Winbrill, Alceste, Hyperbase, Tropes, Lexico...).

12 Aujourd'hui, les logiciels ont gagné en notoriété et en fiabilité, ils mettent à disposition $\mathrm{du}$ chercheur de nombreux outils, en plus des décomptes d'occurrences. Ainsi dans sa contribution centrée sur les professions de foi des politiques, Magali Guaresi montre comment le décompte des cooccurrences permet d'«appréhender les éventuelles modalités genrées de la construction d'identités politiques dans [son] corpus »; de même, 
S. De Sousa interroge les cooccurrences du terme «Etats-Unis » dans le discours révolutionnaire latino-américain, en précisant les mots - et les représentations - qui y sont les plus fréquemment associés. De nombreux calculs statistiques, figures et graphiques très éclairants (voir ces deux contributions) viennent également compléter ces outils. Ces logiciels s'étant répandus hors du cadre des sciences du langage, la période de «méconnaissance [de la plupart] des historiens pour les analyses statistiques des données textuelles et les analyses langagières"(S. De Sousa) semble aujourd'hui dépassée. Cependant, en débordant très largement du cadre de l'AD et du Traitement Automatique des Langues (TAL), l'utilisation de ces logiciels s'éloigne quelquefois des présupposés épistémologiques dans lesquels ils ont été conçus.

13 Ainsi, l'approche méthodologique, répandue en analyse du discours consistant à compléter l'analyse quantitative par une analyse qualitative ou à pratiquer des allersretours entre les deux approches - méthodologie que D. Mayaffre justifie et illustre « pas à pas » dans sa contribution relative à la sur-utilisation du pronom ça par Nicolas Sarkozy -, ne va pas forcément de soi pour les spécialistes de la psychologie sociale, de la sociologie, des sciences politiques ou encore de l'anthropologie qui analysent le langage dans le but d'y lire les pratiques des acteurs et/ou les représentations des sujets ${ }^{5}$. En effet, quelquefois utilisé sans précautions méthodologiques et sans souci des présupposés épistémologiques des sciences du langage et de l'AD, le logiciel semble acquérir une sorte de vertu magique : il dirait de lui-même et par lui-même de quoi est fait le discours. On retrouve alors «l'éternel malentendu » entre $\mathrm{AD}$ et sciences sociales perçu déjà en 1986 par Régine Robin qui dénonçait « une conception transparente de la langue et du discours encore si prévalente dans les sciences humaines aujourd'hui » (Robin 1986 : 128). Ce débat se trouvait d'ailleurs déjà dans l'opposition frontale posée par Pêcheux (1969) entre analyse du discours et « analyses de contenu ».

14 Ce qui est en jeu et qui justifie, me semble-t-il, l'intérêt des réflexions méthodologiques dans lesquelles s'inscrit ce volume, c'est le danger d'assister peu à peu à un recul épistémologique. Des avancées fondamentales, et depuis longtemps actées, comme la remise en question de la bi-univocité du rapport signifiant/signifié et de l'unicité du sujet parlant, les réflexions sur les genres du discours et les styles individuels (voir ici ma propre contribution), sur la constitution du corpus (Mazière 2005), sur la signification des énoncés en contexte, sur la distinction langue/discours, y compris la notion saussurienne de valeur du signe, semblent quelquefois proprement ignorées par certains travaux en ATD. Ainsi assiste-t-on parfois à une méconnaissance des « principes pour l'arbitrage des cas douteux » (Muller 1973 : 9), voire à l'ignorance de l'existence de ces cas douteux ${ }^{6}$.

Afin d'éviter les dérives qui pourraient jeter l'anathème et la suspicion sur l'ensemble des analyses de textes assistées par ordinateur, une réflexion interdisciplinaire sur l'articulation entre les approches quantitatives et qualitatives semble alors s'imposer et c'est l'un des objectifs de ce recueil que d'y contribuer.

\section{Questions de vocabulaire?}

16 L'une des questions traitées dans ce volume concerne les dénominations qualitatif/ quantitatif que tous s'accordent à juger insatisfaisantes.

17 J. Duchastel et D. Laberge montrent ainsi combien certaines oppositions sont "stériles » lorsqu'elles empêchent la recherche de progresser, ils se proposent ainsi d'interroger le 
sens de cette opposition et la place qu'y prend l'AD en offrant un vaste panorama en même temps qu'une vision synthétique des approches et traditions anglo-saxonnes et européennes et des méthodes mixtes qui (co-)existent depuis des décennies. M.-A. Paveau rappelle que les approches qualitatives, jamais vraiment définies, "constituant l'approche non marquée en quelque sorte ", sont considérées comme allant de soi pour nombre d'analystes du discours. Si M. Guaresi estime que «l'opposition [est] désuète entre qualitativistes et quantitativistes ", D. Mayaffre démontre que cette distinction oppositive n'a plus lieu d'être en revenant sur le sémantisme des deux termes et en précisant - rejoignant ainsi J. Duchastel et D. Laberge - qu'il n'existe pas de méthodologies uniquement quantitatives.

S'il est vrai que les approches qualitatives sont définies " en creux " par rapport aux approches quantitatives, il semble également vrai que l'adjectif qualitatives oppose les approches ainsi qualifiées aux "analyses de contenu ». Ces dernières visent surtout à extraire ce qui est dit dans les discours alors que les approches qualitatives s'intéressent en premier lieu à la manière de dire ou encore la « matière discursive » (M.-A. Paveau). La qualité ne s'oppose plus alors à la quantité mais à la teneur, comme on distingue la texture d'un fruit du jus qu'on peut en tirer. De ce point de vue, l'opposition qualitatif/quantitatif ne tient plus puisque les deux approches poursuivent le même but: toutes deux s'attachent en premier lieu à décrire la qualité des textes, ou encore "la texture des textes » (Fairclough 1995), comme on s'attache à décrire la qualité d'un tissu.

Une autre opposition sous-jacente qui fonde l'opposition serait la suivante : le qualitatif permettrait de faire progresser la théorie linguistique, alors que le quantitatif ne se bornerait qu'aux études de cas sans apports théoriques. Si cette opposition peut sembler valide en jetant un regard rapide sur l'histoire de l'analyse du discours et la théorie du discours, elle ne tient plus dès lors qu'on s'intéresse aux possibilités que permet et permettra le traitement de très grands corpus, notamment, comme l'écrit M.-A. Paveau, dans la prise en compte « de l'augmentation des capacités de connaissance humaine » (en termes de mémoire notamment) ou dans le domaine plus restreint de la définition des genres du discours (T. Guilbert).

20 L'argument quasi inverse est quelquefois avancé: les approches quantitatives éloigneraient l'étude de la réalité en allant nécessairement vers plus d'abstraction alors que les approches qualitatives seraient plus proches de la complexité du monde à décrire. Plus qu'un élément distinctif et oppositif entre les deux approches, ce point essentiel est traité ici comme une difficulté ou un écueil à éviter : soit en prenant cette complexité en compte dès la constitution du corpus (T. Guilbert), soit en réduisant puis en restaurant méthodiquement la complexité (J. Duchastel \& D. Laberge).

21 Si la possibilité de généralisation d'une étude - qualitative et/ou quantitative - est discutée par l'ensemble des auteur-e-s, A. Del Ré et R. Nogarini Hilário inscrivent tout particulièrement leur contribution dans ce débat en rappelant certains raccourcis quantitatifs généralisateurs en psychologie sociale et en proposant une forme de généralisation à partir de cas précis d'une population donnée. L'opposition qualitatif/ quantitatif s'appuie ainsi parfois sur l'idée que le quantitatif n'apporterait que des réponses locales et ne justifierait aucune inférence pour d'autres discours - aspect sur lequel insiste D. Mayaffre -, alors que les analyses qualitatives valideraient des hypothèses et produiraient des analyses cumulatives, reproductibles, voire prédictibles. Cette distinction fallacieuse repose, me semble-t-il, sur une commodité de langage non questionnée: la «linguistique de corpus», assimilée aux analyses assistées par 
ordinateur, est opposée communément à la «linguistique théoriciste (sans corpus) ${ }^{7}$ (Rastier 2011: 7). Or, en opposant approches quantitatives (de corpus) et linguistique théorique, on néglige tout un pan de l'AD : on ignore les analyses du discours qualitatives qui travaillent elles aussi sur corpus ou bien on assimile les analyses qualitatives à la linguistique formelle - ce qui revient à nier leur spécificité. Pourtant, la grande majorité des approches qualitatives n'utilisent ni la démarche purement déductive de la linguistique sans corpus, ni les énoncés formels issus du cerveau du chercheur. Approches qualitatives et approches quantitatives sont donc liées à nouveau, en tout cas pour l'ensemble des auteur-e-s contribuant à ce volume, dans le sens où toutes deux s'opposent à la linguistique théorique/formelle par la prise en compte d'un corpus en langage naturel. L'utilisation de démarches hypothético-déductives et/ou inductives fonde, selon D. Mayaffre, la distinction qualitatif/quantitatif; ce point est également discuté par J. Duchastel et D. Laberge. Par ailleurs, il me semble - à l'instar de M.-A. Paveau et A. Del Ré \& R. Hilário - que toute analyse de corpus n'implique pas nécessairement un traitement automatique de celui-ci.

Il y a donc à rétablir ici un «oubli» (au sens de Pêcheux) et à lever une confusion: premièrement, intégrer les approches qualitatives en tant que telles dans les linguistiques de corpus et, deuxièmement, préciser que les études qualitatives n'ont pas moins - ni plus - de vertus cumulatives et reproductibles que les approches quantitatives.

\section{Questions d'ordre méthodologique}

Comment nommer alors ces deux approches? Si les dénominations qualitatif/quantitatif ne sont pas satisfaisantes, cette question reste malgré tout à l'ordre du jour comme le montrent plusieurs contributions - il est indéniable qu'il existe des approches «sans instrument » (M.-A. Paveau) et des approches « avec instrument ». A-t-on affaire à deux méthodologies distinctes et complémentaires? Sans chercher à apporter une réponse dans cette introduction, il semble que l'opposition analyse lexicale/analyse discursive ne soit pas plus pertinente. Si l'on y retrouve le clivage, signalé plus haut, entre les sciences sociales et l'analyse du discours dans l'utilisation des logiciels et des données ${ }^{8}$, les deux approches, avec ou sans instrument, sont lexicales et discursives: d'une part, le traitement assisté par ordinateur n'est plus uniquement lexical depuis longtemps et doit être complété par un retour discursif à l'environnement textuel - voir ici S. De Sousa, M. Guaresi, D. Mayaffre -, d'autre part le traitement «à la main » s'intéresse à la fois aux unités du lexique et aux procédés énonciatifs, argumentatifs, pragmatiques.

Loin de clore le débat en imposant un point de vue unique, les auteur-e-s précisent par une démarche descriptive leur approche méthodologique : soit en montrant par l'exemple les potentialités des logiciels et en justifiant de façon heuristique l'utilisation des outils que ceux-ci mettent à leur disposition (S. De Sousa ; A. Del Ré \& R. Nogarini Hilário ; M. Guaresi), soit en objectivant les différentes étapes de l'analyse d'un discours présidentiel comme autant d'allers-retours entre les deux points de vue qualitatifs et quantitatifs (D. Mayaffre), soit encore en exposant précisément les opérations successives de mise en forme des textes par toute approche mixte et «l'existence [...] d'un arc herméneutique qui ne dissocie pas l'interprétation de l'explication » (J. Duchastel \& D. Laberge), 

d'interpréter un corpus : présupposés épistémologiques de l'AD (T. Guilbert), présupposés contextuels et épistémiques de l'environnement discursif mixte ou des "métissages technolangagiers» (M.-A. Paveau).

aux auteur-e-s, sa mise en pratique, initiée depuis quelques dizaines d'années, nécessite
d'être pensée et étayée par des réflexions à la fois heuristiques et théoriques. C'est ce à
quoi entend contribuer ce numéro de CORELA.

\section{BIBLIOGRAPHIE}

Bacot P., Desmarchelier D. \& Rémi-Giraud S. (éds), 2012. Chiffres et nombres dans

l'argumentation politique. Mots, les langages du politique 100. 5-14.

Barats C. \& Leblanc J.-M., 2013. Généalogie de la construction médiatique du « classement de Shanghai » en France. Corpus de presse et usages sociodiscursifs. Mots, les langages du politique 102. 67-83.

Boutet J. \& Demazières D. (dir.), 2011. Présentation. Langage \& société 135, « Méthodes d'analyse des discours ». 5-8.

Brunet É., 1978. Le Vocabulaire de Jean Giraudoux, structure et évolution. Statistique et informatique appliquées à l'étude des textes à partir des données du Trésor de la Langue Française. Genève: Slatkine.

Brunet É., 2007. Le corpus comme une boule. In F. Rastier et M. Ballabriga (éds), Corpus en lettres et sciences sociales. Des documents numériques à l'interprétation. PUM : Toulouse.

Cos R., 2012. Les élus socialistes face aux chiffres de la délinquance. Dispositions, positions et prises de position partisanes sur les statistiques policières. Mots, les langages du politique, 100. 107-122.

Culioli A., 1968. La formalisation en linguistique. Cahiers pour l'Analyse. 106-117.

Dalud-Vincent M., 2011. Alceste comme outil de traitement d'entretiens semi-directifs : essais et critique pour un usage en sociologie. Langage \& société 135. 9-28.

Dezalay Y. \& Garth B. 1998. Le "Washington consensus". Contribution à une sociologie de l'hégémonie du néolibéralisme. Actes de la recherche en sciences sociales 121-122, 3-22.

Fairclough, N., 1995. Critical Discourse Analysis : The Critical Study of Language. Edimburg: Longman Group Limited.

Fiala P., 1994. L'interprétation en lexicométrie. Une approche quantitative des données lexicales. Langue française 103. 113-122.

Gadet F., 2010. Enjeux de langue dans l'analyse du discours. Semen 29, 111-123.

Garric N. \& Longhi J. (dir.), 2012. Langages 187 « L'analyse de corpus face à l'hétérogénéité de données ». 
Ghiglione R., Matalon B. \& Bacri N., 1985. Les Dires analysés : l'analyse propositionnelle du discours. Presses universitaires de Vincennes.

Guilhaumou J., 2006. Discours et événement : l'histoire langagière des concepts. Besançon : Presses universitaires de Franche-Comté.

Guilhaumou J., 1986. L'historien du discours et la lexicométrie. Histoire et Mesure 1(3-4). 27-46. Groupe Saint-Cloud, 1997. L'image candidate de l'élection présidentielle de 1995. Analyse des discours dans les médias. Paris : L'Harmattan.

Kamber A. (dir.), 2011. Tranel 55, « La linguistique de corpus - de l'analyse quantitative à l'interprétation qualitative ».

Labbé D., 1990. Le vocabulaire de François Mitterrand. Paris: Presses de Sciences Po.

Lafon P., 1984. Dépouillement et statistique en lexicométrie. Paris: Kliensieck.

Lebart L. \& Salem A., 1994. Statistique textuelle. Paris: Dunod.

Léon J., 2010. AAD69 : archéologie d'une étrange machine. Semen 29. 89-109.

Mazière F. \& Guilhaumou J., 2010. Ainsi nous qui sommes dans le Mississipi. Semen 29. 69-88.

Mayaffre D., 2002. L'Herméneutique numérique, L'Astrolabe www.uottawa.ca/academic/ arts/ astrolabe/articles/art0031.htm

Mc Luhan M., 1976 [1964]. Pour comprendre les média. Paris : Seuil, coll. Points.

Muller C., 1973. Initiation aux méthodes de la statistique linguistique. Paris: Champion.

Ogien A., 2013. Désacraliser le chiffre dans l'évaluation du service public. Versailles : Editions Quæ.

Pêcheux M., 1969. Analyse automatique du discours. Paris: Dunod.

Pêcheux M., 1975. Les vérités de La Palice. Paris : Maspero.

Poudat C., 2006. Etude contrastive de l'article scientifique de revue linguistique dans une perspective d'analyse des genres. Thèse de doctorat, Université d'Orléans, http://www.texto-revue.net

Rastier F., 2011. La mesure et le grain. Sémantique de corpus. Paris : Champion.

Robin R., 1986. Postface. L'Analyse du Discours entre la linguistique et les sciences humaines : l'éternel malentendu. Langages 81. 121-128

Salem A., 1993. De travailleurs à salariés. Repères pour une étude de l'évolution du vocabulaire syndical. Mots, les langages du politique 36. 74-83.

Tournier M., 1975. Un vocabulaire ouvrier en 1848. Essai de lexicométrie. Saint-Cloud: Publication de l'École Normale Supérieure.

Vilette M., 2001. Vendre ou se vendre. Notes sur une attitude "libérale" devant la vie. Regards sociologiques 21 . 87-98.

\section{NOTES}

1. Du simple tableur ou traitement de texte à la musique assistée par ordinateur (MAO), la photographie assistée par ordinateur (PAO), la vidéo assistée par ordinateur (VAO)... sans parler $\mathrm{du}$ dessin sur tablettes graphiques, de la conduite automobile ou de l'apprentissage sur ordinateur du pilotage de toutes sortes d'engins.

2. Je reviens rapidement ci-après sur le contexte d'apparition des premiers logiciels. 
3. Ce sentiment est partagé hors de la sphère scientifique, par exemple chez les hommes politiques (Mots, 2012) et chez « de nombreux agents administratifs et policiers » pour qui «[le chiffre de la délinquance générale] demeure encore un référence incontournable " (Cos 2012 : 116).

4. Dans le domaine du sport, par exemple, les statistiques données lors de la diffusion d'un match : nombre de kilomètres parcourus par tel joueur, occupation du terrain, pourcentage de réussite... Mais aussi dans le domaine de l'évaluation : des individus, des politiques publiques, agences de notation des Etats, classements nationaux des lycées ou internationaux des universités (Barats \& Leblanc 2013), etc.

5. Cf. l'argumentaire de la journée d'études précitée, organisée par l'atelier «approches discursives en sociologie » du laboratoire Printemps: «la prise en compte du langage en sociologie dans les études empiriques est une question complexe : les niveaux d'observation, les objets et les méthodes des sciences du langage et des sciences de la société, ainsi que les processus explicatifs ou interprétatifs, ne sont pas a priori les mêmes ".

6. Exemple : grand classé systématiquement comme un adjectif évaluatif ("grand arbre ») alors qu'il peut être axiologique (" grand homme »). Quelle validité accorder aux études s'appuyant sur ces «faits linguistiques»?

7. Autre exemple : «Le développement du traitement automatique des langues, de la statistique et plus généralement de la linguistique de corpus a entraîné... » (Poudat 2006 : 37).

8. La spécificité méthodologique de l'AD au regard des sciences sociales semble résider dans le fait qu'elle prend en compte un certain nombre de présupposés théoriques spécifiques pour la constitution du corpus, l'utilisation des logiciels et l'interprétation des données.

\section{AUTEUR}

\section{THIERRY GUILBERT}

Université de Picardie, CURAPP - UMR 6054 